\title{
Effect of Thermal Treatments on the Schottky Contact between Polycrystalline PbS Thin Film and Gold
}

\author{
ABU Bakara , MUHAMMAD Mansoor b, , MUNEEB Asimc, \\ and SHAHEED Khan ${ }^{\text {d }}$ \\ Institute of Industrial Controls System, Rawalpindi

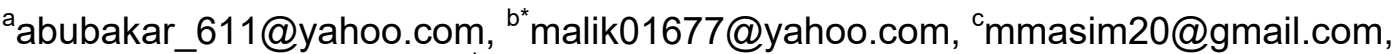 \\ dpe_shaheed@hotmail.com
}

\begin{abstract}
Keywords: PbS Semiconductor Thin Films, Gold Schottky Contacts, IV Testing, Saturation Current, Ideality Factor.
\end{abstract}

\begin{abstract}
Electrical characteristics of semiconductor to metal (S-M) contacts i.e., Schottky contacts, play a vital role towards the performance of the device. The formation of a Schottky contact requires precise thermal treatment, which also affects the electrical characteristic of the junction. In present study, a thin layer of polycrystalline lead sulfide $(\mathrm{PbS})$ was deposited on quartz substrate using chemical bath technique. Metallic electrodes of aluminum and gold were deposited on the each side of the semiconductor film by thermal evaporation method. The aluminum and gold films were $10 \mathrm{~nm}$ and $200 \mathrm{~nm}$ thick, respectively. Latter, the substrates were thermally treated in air for various durations and the effect of the thermal treatments was evaluated by current-voltage (I-V) measurements. It was found that anomalies in the electrical behavior of the $\mathrm{S}-\mathrm{M}$ contacts were mitigated with the treatments. A change from $0.3 \times 10^{-6} \mathrm{~A}$ to $1.3 \times 10^{-6} \mathrm{~A}$ in saturation current was observed. Similarly, the ideality factor (at $V_{t h}<<V_{d}$ ) of the $S-M$ contact was 12 on as deposited contacts, while it reduced to 2.2 after the thermal treatment, which indicates an improved Schottky contact.
\end{abstract}

\section{Introduction}

Among chalcogenides compounds, lead sulfide $(\mathrm{PbS})$ is a IV-VI semiconductor with cubic structure having a direct narrow band gap of $0.41 \mathrm{eV}$ at $300 \mathrm{~K}$, which finds their extensive application in detectors. The spectral region of IR response covers typically 1-3 $\mu \mathrm{m}$ range; making it suitable for infrared applications. This material has also been used in many fields such as photography and solar absorption. Some specific applications include photo resistors, diode lasers, humidity monitors, temperature sensors, and solar control coatings.

An electrical contact is the interface between the current carrying members of electronic devices that assure the continuity of electric circuit. The primary purpose of an electrical connection is to allow the uninterrupted passage of electrical current across the contact interface. It is clear that this can only be achieved if a good metal to semiconductor contact is established. Selection of a contact material depends on various factors like electrical conductivity, work function, electron affinity, resistance to environmental attack, degree of tarnish and ease of processing.

In present work, gold was selected with thin buffer layer of aluminum as contact materials for $\mathrm{PbS}$ semiconductor thin film. The contacts were deposited by thermal evaporation technique. After deposition, the contacts were thermally treated at various temperatures and characterized by using scanning electron microscope (SEM), X-ray diffraction and current-voltage (I-V) testing.

\section{Design of Metal-Semiconductor Contact}

Semiconductor devices, must make contact with the outside world. The contact is made through non-rectifying (ohmic) or rectifying (Schottky) metal-semiconductor junctions. An ohmic contact is a low-resistance junction providing current conduction in both directions, contrarily a Schottky contact generates a conduction barrier between semiconductor and metal junction, which is analogous to a p-n junction [1]. 

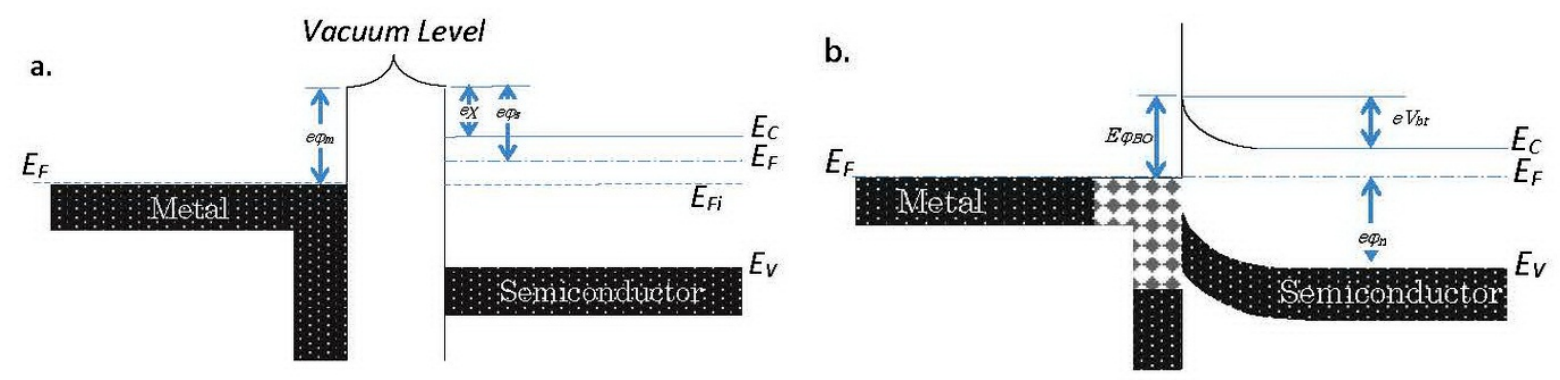

Fig. 1. Ideal energy-band diagram of semiconductor to metal a) before contact; b) after contact.

The ideal energy-band diagram for a particular metal and n-type semiconductor before making contact is shown in Fig. la. The vacuum level is used as a reference energy level. The parameter $\varphi_{\mathrm{m}}$ is the metal work function (measured in $\mathrm{eV}$ ), $\varphi_{\mathrm{s}}$ is the semiconductor work function, and $\chi$ is known as the electron affinity. If we assume that $\varphi_{\mathrm{m}}>\varphi_{\mathrm{s}}$ then the ideal thermal-equilibrium metalsemiconductor energy-band diagram, such a situation is shown in Fig. lb. Before contact, the Fermi level in the semiconductor was above that in the metal. In order for the Fermi level to become a constant through the system in thermal equilibrium, electrons from the semiconductor flow into the lower energy states in the metal. Positively charged donor atoms remain in the semiconductor, creating a space charge region. The parameter $\mathrm{E} \varphi_{\mathrm{BO}}$ is the ideal barrier height of the semiconductor contact: the potential barrier seen by electrons in the metal trying to move into the semiconductor. This barrier is known as the Schotty burrier and is given by[2]:

$$
\mathrm{E} \varphi_{\mathrm{BO}}=\left[\varphi_{\mathrm{m}}-\chi\right]
$$

Eq. 1 was used to calculate the barrier height for PbS-metal Schottky contact for the preset study. It was found in literature that the electron affinity of $\mathrm{PbS}$ is $3.92 \mathrm{eV}$ [3]. To make a Schottky contact; a metal having work function higher than $3.92 \mathrm{eV}$ was required. Therefore, gold was selected as the contact materials owing to the facts that its ease to thermal evaporation, very high corrosion resistance, low tarnishing rate, and high electrical conductance [4]. It has work function of $5.1 \mathrm{eV}$, which can yield a Schottky barrier height up to $1.18 \mathrm{eV}$.

The semiconductor $\mathrm{PbS}$ has face centered cubic (fcc) structure with $5.931 \mathrm{~A}^{\circ}$ lattice parameter. Albeit, gold is also fcc in nature but its lattice parameter is $4.079 \mathrm{~A}^{\circ}$, which makes a much higher difference in the lattice size that will exist at the interface between $\mathrm{PbS}$ and $\mathrm{Au}$. Thin films having higher differences in the lattice parameters usually generate localized stresses in the interface; resulting in localized cracking, high degree of strain and high dislocation density, which may reduce the functionality of electrical contact. Therefore, a buffer layer was suggested between $\mathrm{PbS}$ and $\mathrm{Au}$ to reduce such unwanted stresses. Pure aluminum has fcc structure with a lattice parameter of 4.649 $\mathrm{A}^{\circ}$; therefore a $10 \mathrm{~nm}$ layer of aluminum was suggested between $\mathrm{PbS}$ and $\mathrm{Au}$ films.

\section{Experimental}

Deposition of PbS Thin Films. Detailed methodology and experimentation is discussed elsewhere [5]. Briefly, the lead $\mathrm{PbS}$ films were deposited on glass substrates by the chemical deposition method. Prior to the coating, the glass substrates were washed in methanol and $\mathrm{HCl}$ and finally etched with sulfuric acid. Chemical bath was consisted of thiourea, hydrazine hydrate and lead acetate solution. Glass substrates were mounted horizontally in the bath. Coating temperature was set to $100{ }^{\circ} \mathrm{C}$ and coating was carried out for 30 minutes. After deposition, films were thoroughly washed with demineralized water and dried in oven.

Deposition of Electrical Contacts. For the fabrication of Schottky barrier junction, metal contacts of aluminum and gold were deposited on the $\mathrm{PbS}$ thin film using thermal evaporation technique. Both the coating materials (i.e. $\mathrm{Al} \& \mathrm{Au}$ ) were charged into the fusion boats, which were made of 
tungsten. The glass substrates coated with $\mathrm{PbS}$ film were mounted on the specimen holder along with appropriate mask (Fig. 2a). A vacuum of $4.5 \times 10^{-6}$ mbar and substrate temperature of $100{ }^{\circ} \mathrm{C}$ were maintained in the chamber during the coating. First, a $10 \mathrm{~nm}$ thin coating of aluminum was deposited followed by $200 \mathrm{~nm}$ thick coating of gold as shown in Fig. 2b. The substrates were rotated at constant revolution of $10 \mathrm{rpm}$ during the coating process to reduce the shadowing effects of the mask.

Thermally evaporated coatings generally accompany residual stresses, which may cause spalling and/or cracking of the films. To eliminate such stresses, a thermal annealing treatment was carried out after the coating without breaking the vacuum. The substrate temperature was gradually raised to $200{ }^{\circ} \mathrm{C}$ in two equal intervals i.e. $50{ }^{\circ} \mathrm{C}$ for 30 minutes. Once the annealing temperature was achieved; the substrates were held for two hours followed by slow cooling at the rate of $5{ }^{\circ} \mathrm{C} / \mathrm{min}$ to room temperature.

a

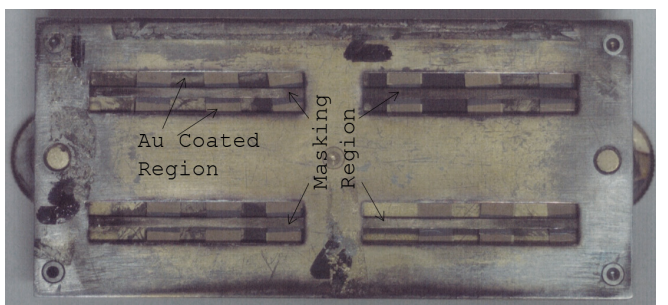

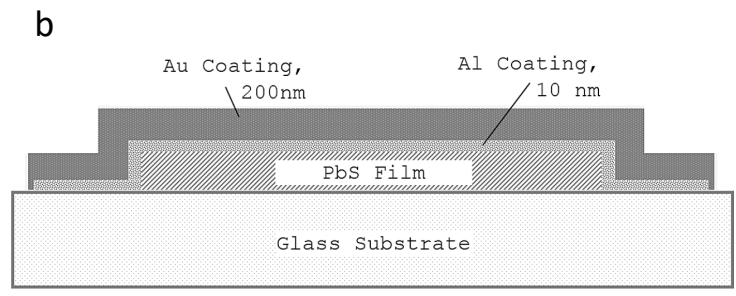

Fig.2. a) Photograph of the masking assembly for the deposition of the contact materials on PbS films, and b) the schematic cross-sectional view of the coatings carried out.

I-V Characteristics. Analysis of I-V characteristics of the Schottky junction allows us to understand different aspects of current transport phenomenon. Therefore, the junction parameters such as ideality factor $(\mathrm{n})$ and saturation current $\left(\mathrm{I}_{\mathrm{s}}\right)$ were calculated from $\mathrm{I}-\mathrm{V}$ characteristics curves of the junctions. All the test were conducted at $25{ }^{\circ} \mathrm{C}$ and $35 \%$ relative humidity.

The IV testing setup comprised of two high precision digital volt and ampere meters with a precision digitally controlled power supply. During the tests, the voltage across the chip was increased gradually from $0-10,000 \times 10^{-3}$ volts, whereas the current drawn was from $0-1.2 \times 10^{-6}$ amperes. The $\mathrm{PbS}$-Au chips were treated thermally at $110{ }^{\circ} \mathrm{C}$ for different durations i.e. $0,30,150$ and 210 minutes and then subjected to IV characterization.

\section{Results and Discussion}

PbS Film. SEM analysis showed the morphology and grain size distribution of PbS crystals in the film. The grains had pyramidal shape with a rather compact surface, as seen in Fig. 3a. The average grain size was sub-micron (i.e. 150 - 450). The deposited PbS films were uniform in nature and had fewer defects like pin-holes, blisters, and discontinues in isolated regions; particularly at the edges of the film.

The XRD pattern, of the deposited PbS films, was measured by using a "JEOL JDX-9C" parallel beam X-ray diffractometer and is shown in Fig. 3b. The pattern revealed polycrystalline structure and all the diffraction peaks were indexed to face centered cubic structure of $\mathrm{PbS}$ compound i.e. (111), (200) and (220), [JCPDS reference no. 78-1901]. However, the integrated peak intensities differed from standard intensities as the results indicate that $\mathrm{PbS}$ thin layers slightly had a preferential orientation at (200) of the grains with c-axis perpendicular to the plane of the glass substrate. 

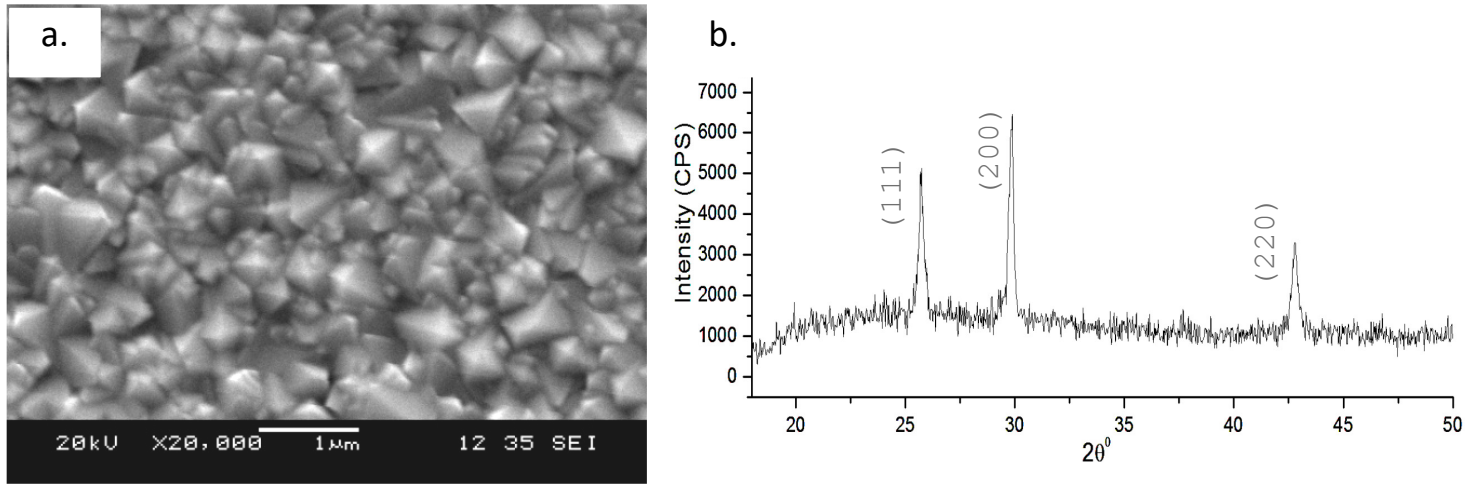

Fig. 3. a)SEM micrograph showing surface morphology of the PbS thin film grown on glass substrate,

b) XRD scan of the film showing the formation of $\mathrm{PbS}$ phase.

Electrical Contacts. As mentioned earlier that the electrical contacts were deposited using thermal evaporation technique. The uniformity and consistency in the deposited layer is preferentially required for substantial electrical characteristics. In Fig. 4, an SEM micrograph is showing the deposited gold film at the top of $\mathrm{PbS}$ layer with a buffer layer of aluminum. It could be seen that the gold film is consistent and adherent to the $\mathrm{PbS}$ film and glass substrate. At higher magnification uniformity of the film is accompanied by some agglomerates as well.
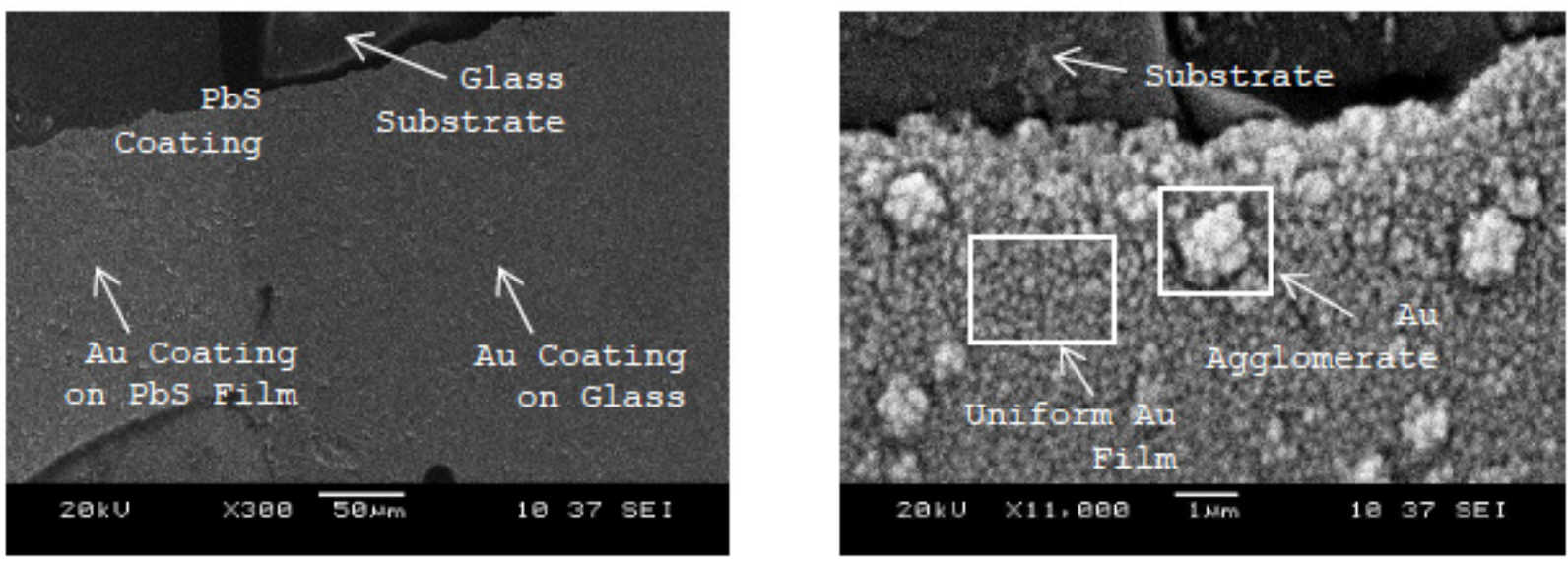

Fig. 4. Thermally evaporated gold film on the PbS substrate.

I-V Characteristics. The superimposed graphs of the I-V testing for the PbS-Au coated chips treated at various temperatures is shown in Fig. 5a.

It was observed that the curve, in case of as deposited ( 0 min.) chip, was lower in current values besides its non-uniform trace. In fact the graph was discontinuous and appeared in steps, whereas the thermal treatments not only removed the stepping features of the curves but also increased the saturation currents with increasing treatment time. Albeit, the increase in saturation and uniformity of the curves were rapidly changed up to 150 minutes with treatment time but it became steady afterwards; a very feeble change was observed at 210 minutes treatment.

The ideality factor (n) of the respective curves was calculated using following equation [6]:

$$
\mathrm{n}=\mathrm{q} / \mathrm{KT}[\delta \mathrm{V} / \delta(\operatorname{lnl})]
$$


a)

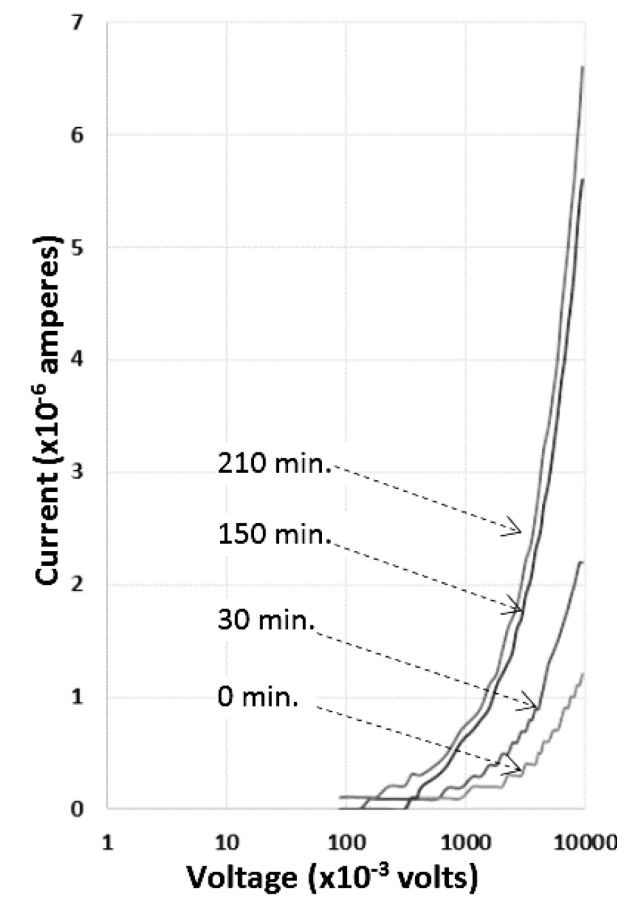

b)

\begin{tabular}{ccc}
\hline $\begin{array}{c}\text { Treatment } \\
\text { Time (min) }\end{array}$ & $\begin{array}{c}\text { Ideality } \\
\text { Factor, n }\end{array}$ & $\begin{array}{c}\text { Saturation } \\
\text { Current, Is } \\
\left(\mathrm{x} 10^{-6} \mathrm{Amp}\right)\end{array}$ \\
\hline 0 & 12 & 0.3 \\
30 & 7.2 & 0.7 \\
150 & 2.7 & 1 \\
210 & 2.2 & 1.3 \\
\hline
\end{tabular}

Fig. 5. a) Superimposed plots of $\mathrm{I}-\mathrm{V}$ characteristics of $\mathrm{PbS}-\mathrm{Au}$ contacts treated at various temperatures, and b) Table showing transport characteristics of the contacts.

The saturation current was determined by drawing the tangential line in the linear section of the individual curves, where the ordinate of the graph was plotted at natural log scale.

It was observed that the ideality factor of the contact was ameliorated from 12 to 2.2; representing a net improvement more than $85 \%[7,8]$. Similarly, the saturation current was also increased from $0.3 \times 10^{-6}$ to $1.3 \times 10^{-6}$, which is almost four times improvement. The higher values of ideality factor were probably due to the potential drop in the interfacial layer and the recombination current through the interfacial states between the semiconductor/metal layers. The other reason for higher values of ideality factor " $n$ " could be attributed to the inhomogeneous barrier height, which became homogeneous with thermal treatments resulting in lowered $\mathrm{n}$ values from 12 to $2.2[3,9]$.

In either case of untreated or treated specimens; the ideality factor remained higher than unity i.e., an indicator of non-ideal behavior of the device, which suggested the transport mechanism(s), other than just thermionic, were probably present in the junctions, which may include:

- the oxide layer grown on the semiconductor, suggesting that the potential barriers at real metal-semiconductor interfaces depends much more the applied voltage than predicted ideal contacts.

- and/or the effect of series resistance due to the presence of an interfacial oxide layer.

\section{Conclusions}

- Gold contacts with aluminum buffer layer were successfully deposited on PbS thin films.

- The gold contacts were uniform and adherent to $\mathrm{PbS}$ and glass surfaces.

- The ideality factor of the contact increased to $>85 \%$ after the treatment of 210 minutes at $110{ }^{\circ} \mathrm{C}$, while the saturation current was increased four times at the same treatment.

\section{Acknowledgement}

Authors are grateful to Mrs. Sabah Zaman for her support and cooperation to the completion of the present work. 


\section{References}

[1] R.G. Streetman, S. Banerjee., Solid State Electronic Devices, 5th ed., Prentice Hall, NJ, 2000.

[2] D.A. Neamen, Semiconductor Physics and Devices: Basic Principles 3rd ed., McGraw-Hill., Boston, 2003.

[3] S. Wang, Fundammrals of Semiconductor: Theory and Devices Physics, Englewoud Cliffi., NJ: Prentice Hall, 1989.

[4] J. Nordstrom, M. Johansson, A. S'anchez Nogueron, E. Calleja, M. Boisen, Investigation of the bond strength between the photo-sensitive polymer SU-8 and gold., Microelectron. Engin., 78-79 ( 2005) 152-157.

[5] S. Zaman, S.K. Mehmood, M. Mansoor, M.M. Asim, Effect of lead ion concentration on the structural and optical properties of nano-crystalline PbS thin films, IOP Conf. Ser. Mat. Sci. Engin., 60 (2014) 012057.

[6] S. Gholami, M. Khakbaz, Measurement of I-V characteristics of a PtSi/p-Si schottky barrier diode at low temperature, Electron. Comm. Engin., 5 (2011) 389-397.

[7] E.H. Rhoderick, R.H. Williams, Metal Semiconductor Contacts, 2nd ed., Clarendon Press, Oxford, 1988.

[8] A. OS, T. Rington, Electrical transport at a non-ideal $\mathrm{CrSi}_{2}-\mathrm{Si}$ junction, Sol. State Electron., 44 (2000) 178-189.

[9] V.L. Rideout, A Review of theTheory,Technology and Applications of Mctal-Semiconductor Rectifiers, Th.Sol. Fil., 3 (1978) 261-291. 